Journal of Patient-Centered

Volume 4

Issue 4 - Health Disparities and Inequities: Part

Article 13 I

$11-6-2017$

\title{
Decreasing Time to Broad Spectrum Antibiotics for Septic Patients in the Emergency Department
}

Joseph J. Zieminski

Emily E. Bryant

Follow this and additional works at: https://aah.org/jpcrr

Part of the Bacterial Infections and Mycoses Commons, Emergency Medicine Commons, Health

Information Technology Commons, Health Services Research Commons, and the Therapeutics Commons

\section{Recommended Citation}

Zieminski JJ, Bryant EE. Decreasing time to broad spectrum antibiotics for septic patients in the emergency department. J Patient Cent Res Rev. 2017;4:257-8.

Published quarterly by Midwest-based health system Advocate Aurora Health and indexed in PubMed Central, the Journal of Patient-Centered Research and Reviews (JPCRR) is an open access, peer-reviewed medical journal focused on disseminating scholarly works devoted to improving patient-centered care practices, health outcomes, and the patient experience. 
Conclusion: Within Aurora, metabolic syndrome is rarely and variably diagnosed in medical practice, despite clear evidence of satisfied ATP III and WHO criteria.

\section{Improved Service Efficiency Improves Racial Disparity in Diabetic Care}

Abel Irena, Kushal Patel, David Thompson, Gregory Schleis, Abiy Gesese, Richard Battiola

\section{Department of Internal Medicine, Aurora Sinai Medical Center; Aurora University of Wisconsin Medical Group}

Background: Racial disparities in diabetes care have been documented. Disparities also have been shown to affect service quality and outcome of diabetic care. Analysis of our internal medicine residency clinic diabetic care management performance across REAL-G (race, ethnicity, age, preferred language and/or gender) showed race-based disparity on two outcome measures: 1) measurement of glycohemoglobin (A1C) at least twice a year; and 2) target blood pressure of $<140 / 90$.

Purpose: Develop interventions to decrease racial disparities in diabetes care among patients managed by an internal medicine residency clinic, as part of the Alliance of Independent Academic Medical Center's National Initiative V project.

Methods: Interventions were developed following analysis of clinic performance data by REAL-G categories, workflow analysis and multidisciplinary clinic team meetings. A point-of-care A1C machine was procured and workflow developed using the Plan-DoStudy-Act cycle. Staff training was conducted. A rolling 12 months data set was obtained from electronic health records. Baseline data range was December 2014 to November 2015, while endline data were from January 2016 to December 2016. The interventions were launched in July 2016. Percentage difference between baseline and endline outcome indicators was calculated and Z-score test assessed. Statistical significance was set at $\mathrm{P}<0.05$.

Results: At baseline, 62.9\% (401 of 638) of patients who selfidentified as African American/black (AA) had A1C measured at least twice a year compared to $74.3 \%$ (107 of 144) of patients who selfidentified as white/Caucasian (WC), a percentage difference of $11.4 \%$ $(\mathrm{P}=0.01)$. For goal blood pressure in diabetics, $71.0 \%$ (453 of 638 ) of AA met the target as compared to $80.6 \%$ (116 of 144) of WC, a percentage difference of $9.6 \%(\mathrm{P}=0.003)$. Following the intervention, a higher percentage of AA patients (71.4\% [381 of 534]) had at least two A1C measured during project period. The outcome also showed improvement for WC (79.8\% [95 of 119]). The percentage difference between races narrowed to $8.5 \%(\mathrm{P}=0.06)$. For goal blood pressure, $75.1 \%$ of AA achieved the target compared to $81.5 \%$ of WC, with percentage difference narrowing to $6.4 \%(\mathrm{P}=0.14)$.

Conclusion: Racial disparities in diabetes were confirmed, even for a clinic setting in which black patients are predominant. Racial disparity can be improved by implementing interventions that improve service for all patients.

\section{Generation of a Patient-Derived Brain Metastasis Breast Cancer Cell Line via Novel Orthotopic Injection Placement and Serial Mouse Transplantation to Develop PDF Mouse Model}

Amber L. LaCrosse, Denise M. Coley, Paul J. Mintz, Santhi D. Konduri, Richard A. Rovin, Amin B. Kassam
Aurora Research Institute; Aurora Neuroscience Innovation Institute

Background: The incidence of brain metastasis appears to be increasing, potentially due to advanced technology that aids early diagnosis. Patient-derived xenografts (PDX) have high translational value, as these models retain key functional characteristics of the patient tumor. PDX models are useful to understand the molecular basis of tumorigenesis and to identify new treatment targets. However, generating a first-line PDX model is challenging as engraftment failure is high. Serial transplanting tumor tissue via mouse-tomouse propagation increases engraftment rates and decreases PDX development time. Herein we report methods to generate a PDX cell line from patient-derived tumor tissue that includes the cerebral aqueduct as a novel intracranial orthotopic implantation site.

Purpose: Develop human tumors in mouse models for therapeutic purpose.

Methods: Patient-derived brain metastasis tumor tissue was enzymatically dissociated into a single cell suspension and maintained in neurocult media supplemented with human recombinant bFGF and EGF $(20 \mathrm{ng} / \mathrm{ml})$. The cells were seeded at a density of $1.0 \times 104 / \mathrm{cm}^{2}$ on ultra-low attachment plates and maintained at $37^{\circ} \mathrm{C}$ with $5 \% \mathrm{CO}_{2}$. PDX models were generated via orthotopic stereotactic surgeries. Athymic nude mice were anesthetized with an intraperitoneal injection of ketamine $(100 \mathrm{mg} / \mathrm{kg})$ and xylazine $(10 \mathrm{mg} / \mathrm{kg})$. The cerebral aqueduct was located using these coordinates from bregma: A: -5 ; L: +0.2 ; V: -2.4 . Mice were injected with $5.0 \times 104$ cells in 2 $\mu \mathrm{l}$ of media at a rate of $0.4 \mathrm{ul} / \mathrm{min}$. Mice were monitored daily for symptoms of tumor formation. Upon becoming symptomatic, mice were euthanized and tumor tissue was harvested for both culture and H\&E stain for tumor verification.

Results: Mice injected with primary patient cells (first-generation mice) developed tumors at 7 weeks (average: 6.77 weeks), secondgeneration mice yielded tumors at 2 weeks (average: 13.5 days), and third-generation mice replicated results from second-generation mice (average: 13 days). H\&E stain revealed invasive tumor masses in the ventricular system that extended from the cerebral aqueduct to the lateral ventricles. Immunohistochemistry analysis confirmed the thirdgeneration cell line retained key characteristics of the patient tumor. Conclusion: These methods successfully generated a PDX cell line from patient-derived brain metastasis that demonstrates reliable tumor formation and phenotypic stability. Importantly, our unique intracranial implantation site revealed several distinct masses, a hallmark of brain metastasis in patients.

\section{Decreasing Time to Broad Spectrum Antibiotics for Septic Patients in the Emergency Department}

Joseph J. Zieminski, Emily E. Bryant

\section{Department of Pharmacy, Aurora St. Luke's Medical Center}

Background: Timely administration of broad spectrum antibiotics has been shown to be directly correlated with decreased mortality for patients with severe sepsis and septic shock. As such, both the Surviving Sepsis Campaign and the SEP-1 CMS measure recognize timely antibiotic administration as a cornerstone of therapy for patients with severe sepsis or septic shock.

Purpose: Decrease time to broad spectrum antibiotic administration for septic patients in the emergency department (ED) of Aurora St. Luke's Medical Center.

Methods: An alert within the electronic medical record was created to more rapidly identify potentially septic patients in the ED. After 
receiving the alert, ED pharmacists reviewed the patient profile, including differential diagnosis; antibiotic allergies, reactions and/ or previous tolerance; recent bacterial cultures; and any antibiotics already ordered, to assess dose and spectrum of coverage. Pharmacists intervened as needed to ensure patients received appropriate broad spectrum antibiotics. Antibiotics were defined as broad spectrum in the same manner as by the Centers of Medicare and Medicaid Services in its Early Management Bundle, Severe Sepsis/Septic Shock. Education was provided to physicians, nurses and pharmacists to encourage timely administration. Outcomes to be measured include mean time to broad spectrum antibiotics, percentage of patients who received broad spectrum antibiotics within 1 hour of presentation, and percentage of patients who received broad spectrum antibiotics within 3 hours of presentation. Results: Pre-alert intervention, patients who were coded with the ICD-10 codes for sepsis, severe sepsis or septic shock in May $2016(n=65)$ were analyzed to determine mean time to broad spectrum antibiotics $(2.81 \pm 1.63$ hours $)$, percentage of patients who received broad spectrum antibiotics within 1 hour $(3.1 \%)$, and percentage of patients who received broad spectrum antibiotics within 3 hours $(67.7 \%)$. Post-alert outcomes will be reported at Aurora Scientific Day.

Conclusion: To be reported at Aurora Scientific Day.

\section{Mechanisms of Left Ventricular Thrombus Formation in Heart Failure With Reduced Ejection Fraction: Novel Insights From Two-Dimensional Speckle Tracking Echocardiography}

Dhruv Chawla, Tracy Hammonds, Tadele Mengesha, Matt Umland, Khawaja Afzal Ammar, Vinay Thohan

\section{Aurora Cardiovascular Services; Aurora Research Institute}

Background: Patients suffering from heart failure with reduced ejection fraction (HFrEF) are at increased risk for left ventricular (LV) thrombus and subsequent thromboembolism, yet anticoagulation is not routinely recommended for left ventricular ejection fraction (LVEF) alone. We sought to determine the role of two-dimensional speckle tracking echocardiography (2D-STE) to quantify regional changes in cardiac function associated with LV thrombus, which may prospectively guide anticoagulation.

Purpose: Help enable cardiovascular clinicians to use 2D-STE to evaluate regional strain patterns among patients with HFrEF with and without LV thrombus. Our results suggest that statistically lower regional longitudinal strain patterns in a well-matched cohort identified patients with thrombus. The postulate that these patterns existed prior to the formation of thrombus remains to be tested. Our results warrant further investigation with a larger prospective cohort. Methods: We retrospectively identified patients with $\mathrm{LVEF} \leq 35 \%$ who had LV thrombus $(\mathrm{n}=12)$ and a matched (in demographics and LVEF) cohort who did not have LV thrombus $(n=36)$. We performed offline 2D-STE longitudinal strain analysis. Descriptive statistics were used to compare variables.

Results: The average age of identified patients was $62.7 \pm 15.0$ years; $71 \%$ were male. LVEF was not statistically different. LV enddiastolic diameter was increased in LV thrombus group (6.2 \pm 1.1 $\mathrm{cm}$ vs $5.5 \pm 0.65 \mathrm{~cm}, \mathrm{P}=0.014)$. $\mathrm{LV}$ thrombus group had consistently reduced regional strain in the inferior wall $(-5.3 \pm 3.9$ vs $-8.3 \pm 4.1$, $\mathrm{P}=0.033)$, at the apex $(-5.3 \pm 4.4$ vs $-8.9 \pm 4.0, \mathrm{P}=0.012)$, and in the left anterior descending coronary distribution $(-5.3 \pm 3.4$ vs $-7.8 \pm 3.4, \mathrm{P}=0.031)$. There was no significant difference in global longitudinal strain or strain dispersion.
Conclusion: Among patients with HFrEF, LV thrombus is associated with reduced longitudinal strain in inferior and apical regions and in the left anterior descending territory.

\section{Challenges in Delivering Refugee Health Systems}

Thy Vo, Fabiana Kotovicz

University of Wisconsin School of Medicine and Public Health; Department of Family Medicine, Aurora University of Wisconsin Medical Group

Background: Aurora Health Care is the major health care system providing care to refugees in Milwaukee, where half of Wisconsin's refugee population resides. Like many other institutions caring for refugee patients, Aurora faces significant challenges when trying to address refugee health needs. Even with the assistance of medical interpreters, cultural differences, language barriers and limited patient health literacy, as well as lack of knowledge of refugee patients' backgrounds, are major obstacles encountered by health care providers in this setting.

Purpose: This quality improvement study aims to assess Aurora providers' perceptions of the benefits and barriers to working with refugee patient populations.

Methods: An online survey was distributed to health care providers and staff at two academic family practice clinics before and after a 5-session educational series. Each educational session delivered monthly focused on refugee cultural awareness. Mental health providers and former refugees working as case managers or interpreters provided education about the main refugee populations in Wisconsin and the refugee resettlement process. The survey assessed participants' perceptions about providing health care to refugees. Participants were asked to respond to questions on a Likert scale from 1 to 7 (ie, strongly disagree to strongly agree) and to two questions comprised of rank choices investigating barriers to effective health care delivery. Responses to questions on Likert scale $\geq 5$ were lumped into an agreement category, while all others were considered a disagreement. Fisher's exact test was used to compare pre- and posteducation responses.

Results: Perception about new medical knowledge and cultural competency had statistically significant increase comparing pre- and postintervention data $(\mathrm{P}=0.0474)$. Insufficient interpreter services and insufficient time for appointments were ranked the top barriers to providing health care services to refugees before and after intervention. Participants also ranked refugees' poor understanding of the U.S. health care system as the biggest challenge in delivering care for refugee patients before and after intervention, followed by communication with resettlement agencies in the preintervention survey and cultural differences in the postintervention survey.

Conclusion: Promotion of cultural awareness and proficiency within groups delivering primary care to refugees can be a valuable tool when trying to overcome obstacles.

Identifying and Targeting Age-Related Colorectal Cancer Screening Rate Disparities in Family Medicine Residency Clinics

Jonathan J. Blaza, Jasmine R. Wiley, Matthew Gill, Alonzo Jalan, Will Lehmann, Deborah Simpson, Jeffrey A. Stearns

Departments of Family Medicine and Academic Affairs, Aurora University of Wisconsin Medical Group 